\section{Caveat emptor!}

John Postgate

Encyclopedia of Microbiology. Edited by Joshua Lederberg. Academic: 1992. Four volumes (pp. 648, 646, 642 and 582). £450, $\$ 695$.

THIS is not an encyclopaedia at all. By definition - and also according to bibliographic usage - an encyclopaedia must be complete: a comprehensive repository of learning, even if today some restriction on the disciplines covered is acceptable. Complete coverage is not attempted here. Although the preface declares that the work's objective is to "survey the entire field [of microbiology] coherently" it then, contradicting itself, describes its contents as "complementing material that would be included in ... university study".

The work comprises more than 200 review-type articles on diverse aspects of microbiology, intended to be "accessible to talented high school and college students" as well as to teachers, technicians and research specialists. The articles do not include reference citations, but end with recommendations for further reading. Useful adjuncts are a preface and guide to microbiological journals (repeated at the beginning of each volume); a glossary of relevant specialized terms (at the head of each article); and a list of contributors, a regular index, a cross-index bringing related titles together, and linkage maps of Bacillus subtilis, Escherichia coli and Salmonella typhimurium (all at the end of Volume 4). The two-column layout is easy on the eye; diagrams are good, but reproduction of photographs is so-so.

The variety of topics covered by the contributors is indeed eclectic; here is a selection: acetogens, biomonitors, catabolite repression, ELISA, food biotechnology, Gram-positive cocci, hepatitis, interferons, laboratory safety, "Method, philosophy of", nucleotide metabolism, origin of life, phylogeny, recA, sulphur cycle, $\mathrm{T}$ cells, ureases, new viruses, wine. Obviously it would take an unconscionable time to assess each of the 200 or so articles critically, and I have not attempted to do so, although I have browsed through most and read some 70 carefully.

Their accuracy, as far as I can judge, is generally impeccable, and the level of clarity is commendably high: I had no serious difficulty with wholly unfamiliar aspects of the subject. Indeed, a few, such as those on influenza and ribosomes, are models of presentation. The majority are useful, workman-like surveys, but several are too focused on the United States to be of more than parochial use (such as those on careers in microbiology, guidelines for genetic manipulation, and, especially inexcusable in view of its African dimension, AIDS). One or two are on the borderlines of microbiology (plant cell culture, vertebrate tissue specificity). Some articles are more verbose and convoluted than others, and there are those that irritate the specialist because of strange omissions or emphases - but unevenness of that kind is to be expected in a multi-authored work.

I found few misprints and misspellings, but some evidence of indifferent editing. One is inured to 'data' and 'none' appearing indiscriminately as singular or plural nouns, but what editor could allow the contributor on bacterial adhesion consistently to use "flagellae" as the plural of flagellum? (Happily the definitive article on flagella gets it right.) A conflict between the equation for nitrogenase action under 'nitrogen cycle' and that in the very next article, on nodules, ought to have been resolved (the second is correct). And some contributors have been so accustomed to using reference citations in their texts that they have not adjusted to their absence, so we find, in one piece, a useless allusion to an anonymous "the same author"; in another, mention of the views of one Brian Spratt, with no further provenance.

However, the work's most serious fault, given its title, remains the lack of encyclopaedic coverage. For example, the only article specifically about antibiotics deals with microbial resistance to them, and although they do turn up in other contexts (under antifungals, ribosomes, biopharmaceuticals and so on), there are no coherent accounts of their production, properties, modes of action, biological significance and requirement by certain mutants. Again, the article on chemotaxis is first-class, but phototaxis, magnetotaxis and less-studied tactic responses are not dealt with. And yet again, the physiology of starvation is wholly ignored, in favour of admittedly fascinating recent work on starvation stress proteins. These are but three of many gaps I noticed.

Information transfer in science is approaching a major discontinuity, as the electronic revolution of the $1980 \mathrm{~s}$ develops and busy researchers cease to bother with anything that is too old to have got into the computer databases. Therefore, if only as a springboard for an on-line future, a proper encyclopaedia of microbiology would have been of tremendous value. This is no such thing. Yet regarded as a grand compendium of essay-reviews dealing with aspects of modern microbiology, the book is a wide-ranging and often absorbing companion to regular textbook mat- erial, especially for US nationals. Why, then, has it been so misleadingly named? I trust it is simply that the editorial board was muddled, as the contradiction in the preface suggests, and not a matter of commercial chicanery.

John Postgate is emeritus professor of microbiology at the University of Sussex, Brighton BN1 9RQ, UK.

\section{Childhood}

\section{matters}

\section{Andrew Whiten}

Beyond Modularity: A Developmental Perspective on Cognitive Sclence. By Annette Karmiloff-Smith. MIT Press: 1992. Pp. 234. \$34.95, £27.50.

"I HATE babies!" the cognitive scientist inside the author's head wants to shout, railing against the tribute paid to one of her lectures by a student clearly swayed by the cuteness of her subject - the human infant. Karmiloff-Smith goes on to reassure us that such irritation is not what she feels as a parent, but the welling outburst illustrates graphically a central intention of the book: "to treat cognitive development as a serious theoretical science contributing to the discussion of how the human mind is organised internally, and not as merely a cute empirical database about when external behaviour can be observed."

The study of development is thus a means to an end, rather than an end in itself. It is advocated as a tool with a unique part to play in establishing the nature of the human mind. In recent years, the computational techniques that help to define 'cognitive science' have been directed at understanding aspects of developmental psychology: here, Karmiloff-Smith aims to turn the tables and explore what developmental research can contribute to cognitive science as a whole. The extent to which she can be judged to have succeeded depends on the scope of 'human mind' that developmental study is claimed to illuminate.

If this is the adult mind, as KarmiloffSmith implies in some parts of the text, then the significance of development must lie solely in its legacy to this mature state. The adult mind will have suchand-such an architecture because the developmental processes used to build it are of a certain character. This is an ambitious claim and the data marshalled here explicitly to support it seem to be relatively few so far, although clearly of great theoretical importance (E. Spelke's work on object perception 